Résumés des conférences et travaux

\title{
Syntaxe indo-européenne
}

\section{Olav Hackstein}

\section{(2) OpenEdition Journals}

Édition électronique

URL : https://journals.openedition.org/ashp/2016

DOI : $10.4000 /$ ashp.2016

ISSN : 1969-6310

Éditeur

Publications de l'École Pratique des Hautes Études

\section{Édition imprimée}

Date de publication : 1 septembre 2017

Pagination : 430-432

ISSN : 0766-0677

\section{Référence électronique}

Olav Hackstein, «Syntaxe indo-européenne », Annuaire de l'École pratique des hautes études (EPHE), Section des sciences historiques et philologiques [En ligne], 148 | 2017, mis en ligne le 04 octobre 2017, consulté le 06 juillet 2021. URL : http://journals.openedition.org/ashp/2016 ; DOI : https://doi.org/ 10.4000/ashp.2016 


\title{
SYNTAXE INDO-EUROPÉENNE
}

\author{
Conférences de M. Olav HaCKstein, \\ université de Munich, \\ directeur d'études invité
}

La série de conférences a pour sujet précis : Négation, préverbe et univerbation en indo-européen : dynamique historique et reconstruction. Les conférences ont porté sur la dynamique diachronique de la négation en indo-européen (place et sens de la négation, négation et interrogation), et sur la reconstruction des préverbes (place et composition verbale). Deux processus diachroniques qui accompagnent l'évolution de la négation et des préverbes sont traités en détail : l'univerbation (y compris le processus de coalescence, en anglais chunking), et la conversion de syntagme ou de phrase, à savoir la transformation de celles-ci en particules et en mots fonctionnels. Une meilleure compréhension de ces processus est susceptible d'enrichir les méthodes de la reconstruction syntaxique.

\section{Négation et interrogation en indo-européen}

La conférence a présenté une synthèse des recherches sur l'étymologie, la syntaxe et le sens des négations en proto-indo-européen, cf. Dunkel 2014, t. II, p. 530 sq. Une attention particulière es accordée à l'emploi des négations dans les phrases interrogatives, spécialement pour les interrogations totales (cf. Hackstein 2013) et les interrogations causales. On constate dans diverses langues l'évolution des négations en particules affirmatives, en conjonctions et en complémenteurs. Plusieurs études d'exemples sont présentées, ce qui inclut la reconstruction morphologique et syntaxique de tous les processus impliqués. En outre, on a traité de la question du comportement des négations dans l'univerbation (e.g. lat. nolō, neglegere, russe nenavidet', cf. Dunkel 2014, t. II, p. 540 sq.).

\section{Préverbes et composés verbaux en indo-européen}

La deuxième conférence a présenté une synthèse des recherches sur la syntaxe des préverbes séparables en proto-indo-européen et le succès croissant de la composition verbale dans les différentes langues indo-européennes, au cours de leur développement séparé. Un nombre important de combinaisons préverbe + verbe peuvent être reconstruites pour le proto-indo-européen, voir le bilan le plus récent dans Dunkel 2014. La conférence a montré comment la composition verbale s'est développée en indo-européen tardif, à partir d'une situation où les préverbes étaient séparés. On examine à nouveaux frais la dichotomie entre préverbes séparables et inséparables en germanique occidental. 


\section{Univerbation et reconstruction syntaxique}

La troisième conférence a traité du mécanisme diachronique de l'univerbation et des facteurs qui favorisent cette univerbation. Dans la bibliographie récente sur la grammaire des emplois («Usage-Based Grammar»), plusieurs processus qui accompagnent l'univerbation et la formation de morphèmes complexes (alias coalescence, angl. chunking) ont été identifiés, précisément : sur le plan sémantique, la perte de la compositionalité, sur le plan phonologique l'usure et la réduction formelles, sur le plan syntaxique le figement de structures morphologiques et syntaxiques. Pour une vue d'ensemble à partir du proto-indo-européen, voir Hackstein 2012. La capacité conservatoire de l'univerbation et de la coalescence fournit des points d'appui importants à la reconstruction syntaxique, aussi bien au niveau de la phrase que du syntagme, e.g.

$1 \mathrm{PN}>\mathrm{Adv}$ : lat. perendie; cottidie; forti mente $>\rightarrow$ fr. fortement;

$2 \mathrm{~S}>\mathrm{N} /$ Adj : lat. necesse (cf. Hackstein 2014b, p. 135-138);

La conférence a présenté des études de divers cas, y compris la reconstruction morphologique et syntaxique des exemples ci-dessus.

\section{Fusion de phrase en indo-européen. De la phrase à la particule, à la conjonction et au complémenteur}

La quatrième conférence a envisagé la conversion de phrases en particules, conjonctions et complémenteurs en indo-européen. On en trouvera un aperçu dans Hackstein 2004a, 2004b. Les structures originelles sont des propositions principales (alias Matrix Clauses $=\mathrm{MC}$ ), ce qui comprend les types principaux de phrase (déclarative, impérative, interrogative) et les propositions subordonnées (alias Subordinate Clauses $=\mathrm{SC})$, voir les exemples suivants :

1a $\mathrm{S}(\mathrm{MC}:$ déclarative $)>\mathrm{P}:$ e.g. russe net (particule négative interactive);

$1 \mathrm{~b} \mathrm{~S}(\mathrm{SC}:$ conditionnelle) $>\mathrm{P}:$ e.g. allemand nur (particule restrictive);

$2 \mathrm{~S}(\mathrm{MC}:$ impérative $)>\mathrm{P}:$ e.g. latin cedo (particule affirmative);

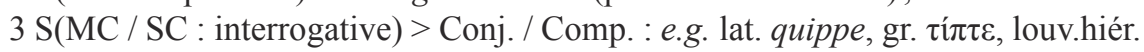
kwipa (particule explicative ou causale).

La conversion de phrases interrogatives (à travers la forme des questions rhétoriques) en particules, conjonctions et complémenteurs est largement attestée, et d'une grande importance. Les expressions interrogatives sont des outils stratégiques pour établir la cohérence textuelle. Il n'est donc pas surprenant que les interrogatifs soient une source fréquence de coordinations adverbiales, de subordonnants et de complémenteurs. La conférence a traité des cas correspondant aux trois catégories énumérées ci-dessus. Le passage d'interrogatif à conjonction a fait l'objet d'une attention particulière.

\section{Références bibliographiques}

Dunkel (George E.) 2014. Lexikon der indogermanischen Partikeln und Pronominalstämme. Band 1: Einleitung, Terminologie, Lautgesetze, Adverbialendungen, Nominalsuffixe, Anhänge und Indices, Band 2: Lexikon, Heidelberg. 
Hackstein (Olav) 2004a. « Rhetorical questions and the grammaticalization of interrogative pronouns as conjunctions in Indo-European », dans Adam Hyllested, Anders Richard Jørgensen, Jenny Helena Larsson et Thomas Olander (éd.), Per Aspera Ad Asteriscos, Studia Indogermanica in honorem Jens Elmegård Rasmussen sexagenarii Idibus Martiis anno $M M I V$, Innsbruck, Innsbrucker Beiträge zur Sprachwissenschaft, p. 167-186.

Hackstein (Olav) 2004b. " From discourse to syntax: The case of compound interrogatives in Indo-European and beyond », dans Karlene Jones-Bley et al. (éd.), Proceedings of the Fifteenth Annual UCLA Indo-European Conference (Los Angeles, November 7-8, 2003), Washington D.C., Institute for the Study of Man (JIES Monograph Series, 49), p. 256-298.

Hackstein (Olav) 2012. "When words coalesce: chunking and morphophonemic extension », dans H. Craig Melchert (éd.), The Indo-European Verb. Proceedings of the Conference of the Society for Indo-European Studies (Los Angeles, 13-15 September 2010), Wiesbaden, Reichert Verlag, p. 87-104.

Hackstein, (Olav) 2013. « Polar questions and non-headed conditionals in a cross-linguistic and historical perspective », dans Benjamin Slade et Shu-Fen Chen (éd.), Grammatica et verba. Glamor and verve. Studies in South Asian, Historical, and Indo-European Linguistics in Honor of Hans Henrich Hock on the Occasion of His Seventy-Fifth Birthday, Ann Arbor, New York, Beech Stave Press, p. 99-116.

Hackstein (Olav) 2014a. « Univerbierung und irreguläre Reduktion in temporalen Adverbien. Uridg. ges-tern von Bopp bis heute », dans Craig Melchert, Elisabeth Rieken et Thomas Steer (éd.), Munus amicitiae Norbert Oettinger a collegis et amicis dicatum, Ann Arbor, New York, Beech Stave Press, p. 32-45.

Hackstein (Olav) 2014b. " Der negative Existentialsatz im Indogermanischen », Historische Sprachforschung 125, 2012 [2014], p. 123-142. 